\title{
Magnetic field induced inversion in the effect of particle size on powder cohesiveness
}

Cite as: J. Chem. Phys. 133, 024706 (2010); https://doi.org/10.1063/1.3458002

Submitted: 16 March 2010. Accepted: 04 June 2010 . Published Online: 14 July 2010

M. J. Espin, J. M. Valverde, M. A. S. Quintanilla, and A. Castellanos

\section{ARTICLES YOU MAY BE INTERESTED IN}

Magnetorheological effect in the magnetic field oriented along the vorticity

Journal of Rheology 58, 1829 (2014); https://doi.org/10.1122/1.4893586

Rheology of magnetofluidized fine powders: The role of interparticle contact forces

Journal of Rheology 54, 719 (2010); https://doi.org/10.1122/1.3380851

Magnetorheology of magnetic holes compared to magnetic particles

Journal of Rheology 44, 91 (2000); https://doi.org/10.1122/1.551075
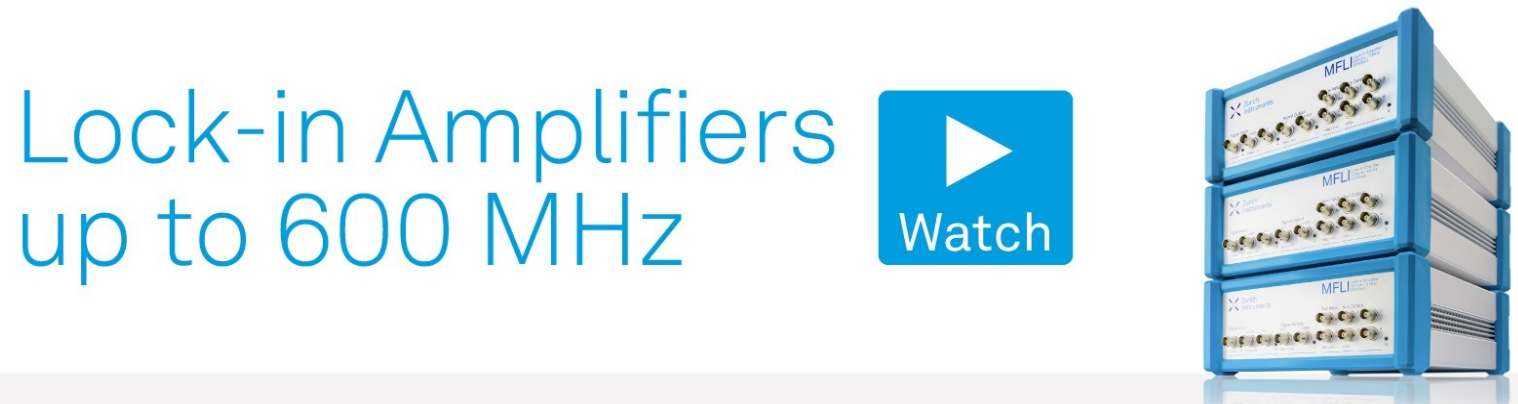

J. Chem. Phys. 133, 024706 (2010); https://doi.org/10.1063/1.3458002

133,024706

(c) 2010 American Institute of Physics. 


\title{
Magnetic field induced inversion in the effect of particle size on powder cohesiveness
}

\author{
M. J. Espin, ${ }^{1}$ J. M. Valverde, ${ }^{2, a)}$ M. A. S. Quintanilla, ${ }^{2}$ and A. Castellanos ${ }^{2}$ \\ ${ }^{1}$ Department of Applied Physics II, University of Seville, Avenida Reina Mercedes $s / n, 41012$ Sevilla, Spain \\ ${ }^{2}$ Department of Electronics and Electromagnetism, University of Seville, Avenida Reina Mercedes $s / n$, \\ 41012 Sevilla, Spain
}

(Received 16 March 2010; accepted 4 June 2010; published online 14 July 2010)

\begin{abstract}
Experimental measurements are reported on the tensile yield stress of magnetofluidized beds of fine magnetic powders operated in the cross-flow configuration. In the absence of externally applied magnetic field the yield stress of the powder depends on particle size as expected, i.e., it increases as bead size is decreased. This trend is however inverted when an external magnetic field is applied. It is suggested that the average orientation of interparticle contacts relative to the direction of the field as affected by particle size plays a relevant role on the magnetic yield stress of these systems. () 2010 American Institute of Physics. [doi:10.1063/1.3458002]
\end{abstract}

The mechanics of magnetizable particle beds is strongly influenced by the presence of magnetic fields, which is the basis of many technological applications. Magnetorheological fluids (MRFs), usually employed as dampeners in many industries, consist of suspensions of magnetizable micronsized particles in nonmagnetic oil-based liquids of similar density to offset sedimentation. ${ }^{1}$ In magnetofluidized beds (MFBs) the magnetic particles are suspended by an upward flowing gas. Generally the application of a magnetic field to these systems induces strong interparticle interactions, which leads to a reversible transition from a liquidlike behavior to a solidlike one characterized by the appearance of a yield stress. The jamming transition in MFBs induced by the magnetic field serves to suppress gas bubbles, thus increasing the gas-solid contact efficiency, which is of interest for reaction catalysis. ${ }^{2}$ The control and transport of magnetic particles is a focus in diverse industries such as xerography ${ }^{3}$ and for medical applications as, for example, controlled drug delivery by means of tailored magnetic nanoparticles. ${ }^{4}$ The flow and structuring of magnetic particles is also a concern in geological studies where palaeoflow directions in volcanic rocks and sediments are usually determined by the effect of existing magnetic fields. ${ }^{5}$ In this work we focus our attention on the yield stress of MFBs and, particularly, on the effect of particle size.

Essentially, a magnetic field acting upon a bed of magnetic particles causes particle magnetization, which gives rise to interparticle attractive forces. As the strength of interparticle forces is increased particle chains become eventually jammed. In the case of linearly magnetizable materials of permeability $\mu_{p}$ surrounded by a fluid of permeability $\mu_{f}$, the magnetostatic force between two isolated particles $\mathbf{f}_{m}$ can be calculated by means of a multipolar expansion, ${ }^{6}$

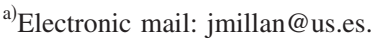

$$
\mathbf{f}_{m}=f_{m}^{0}\left(\frac{d_{p}}{r}\right)^{4}\left[\left(2 f_{\|} \cos ^{2} \theta-f_{\perp} \sin ^{2} \theta\right) \hat{u}_{r}+f_{\Gamma} \sin 2 \theta \hat{u}_{\theta}\right],
$$

where $f_{m}^{0}=(3 / 16) \pi \mu_{f} d_{p}^{2} \beta^{2} H^{2}, H$ is the strength of the externally applied magnetizing field, $\beta=\left(\mu_{p}-\mu_{f}\right) /\left(\mu_{p}+2 \mu_{f}\right), r$ is the distance between the particle centers, and $\theta$ is the angle between the particle chain and the field. The force coefficients $f_{i}$ can be calculated in terms of the multipole moments ${ }^{7}$ and depend on $\alpha=\mu_{p} / \mu_{f}$ and $r$, being $f_{i}=1$ in the dipolar approximation $\left(r / d_{p} \rightarrow \infty\right)$ and in the limit $\alpha \rightarrow 1$. As the permeability of the particles is increased the attractive interaction is further enhanced. For example, in the case of two spheres at contact $\left(r / d_{p}=1\right)$ of $\alpha=10$, it is $f_{\|}=7.287$, $f_{\perp}=0.6192$, and $f_{\Gamma}=1.5035 .{ }^{\gamma}$ In Fig. 1 it is plotted the magnetostatic force between two spheres that we have numerically calculated by means of three-dimensional (3D) finite element method (FEM) ${ }^{8}$ where the Maxwell stress tensor is integrated over the bead surfaces, and the analytically calculated force from Eq. (1) $(\alpha=10)$. The numerical calculation of the fields shows that in the region of the spheres close to the contact the local magnetization field is not aligned with the external magnetic field, as demonstrated in the inset of Fig. 1, showing that magnetization is intensified and tends to point along the normal of the interparticle contact surface, thus giving rise to a strong north-to-south attractive forces between the spheres. The influence of the induced magnetic force on the cohesive behavior of beds of magnetized particles has been brought to light by several experimental works. For example, Forsyth et al. ${ }^{9}$ showed that the void fraction of a bed of magnetic spheres poured into a container in the presence a magnetic field increased linearly to the ratio of interparticle magnetic force to particle weight. Likewise, experimental results reported by Peters and Lemaire ${ }^{10}$ demonstrated that the maximum angle of stability of a tilted bed of magnetic spheres subjected to a magnetic field was significantly increased. In these systems, however, the interaction of the magnetic particles with the surrounding fluid was 


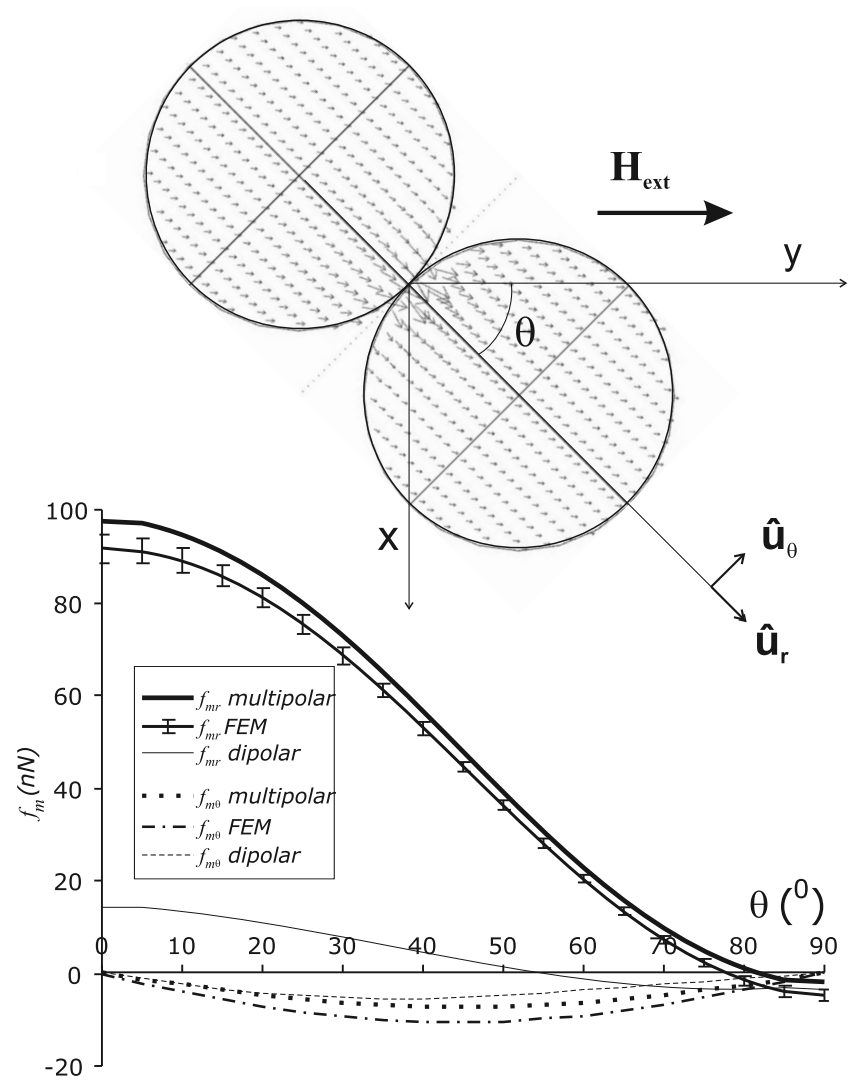

FIG. 1. Top: magnetization field lines numerically obtained for two spheres $(\alpha=10)$ of diameter $d_{p}=35 \mu \mathrm{m}$ separated by a distance $s=0.001 d_{p}$ and subjected to an external field of strength $H=3 \mathrm{kA} / \mathrm{m}$. Bottom: normal $\left(f_{r}\right)$ and tangential $\left(f_{\theta}\right)$ components of the magnetostatic force between the two spheres as a function of the angle $\theta$ that forms the line between the center of the spheres with the external $\mathbf{H}$ field. Forces calculated by means of the dipolar approximation, multipolar analytical expansion, and numerical 3D FEM are shown. Error bars indicate the indeterminacy due to numerical convergence tolerance.

negligible. This two-phase interaction is a main ingredient in the behavior MRFs and MFBs, which will be the focus of the present paper.

The magnetic stress that arises from the interparticle magnetic force $f_{m}$ when a fluidized bed of magnetized particles is jammed will be $\sigma_{m} \propto N f_{m} / S$. Here $N / S$ is the number of particle chains per unit surface that should scale as $\phi / d_{p}^{2}$ for any type of microstructure, where $\phi$ is the particle volume fraction. Thus, $\sigma_{m}$ should be independent of particle size. The same result may be obtained from a simple dimensional analysis. ${ }^{2}$ Since the magnetostatic contact force between particles depends basically on particle magnetization $M_{p}$ and particle size, and the coordination number (average number of contacts per particle) depends on $\phi$, a functional relationship $\sigma_{m}=f\left(\mu_{0}, M_{p}, d_{p}, \phi\right)$ can be postulated. By assuming a power-law relationship, the dimensional analysis leads to $\sigma_{m}=f(\phi) \mu_{0} M_{p}^{2}$, where $f(\phi)$ is a function depending on the type of microstructure. Some experimental observations made on MRFs show instead that the size of the particles can strongly change the magnetic yield stress. ${ }^{11-13}$ Lemaire et al. ${ }^{12}$ reported on a large increase of the yield stress with $d_{p}$ for monodisperse samples of magnetite beads in the size range $0.5 \mu \mathrm{m}<d_{p}<1 \mu \mathrm{m}$. Despite that the ratio $\lambda$ of magnetostatic energy to thermal energy was rather large $(\lambda$ $\approx 10^{2}-10^{3}$ ), Lemaire et al. rationalized this unexpected result from the effect of Brownian fluctuations of the particle positions in the chains. Accordingly, they found that the yield stress was insensitive to particle size for larger particle diameters (between 25 and $45 \mu \mathrm{m}$ ). de Gans et al. ${ }^{13}$ found the same particle effect but correlated it with the average chain length as evidenced by microstructure images, showing chain formation by the largest particles but poor structuring in the case of the smallest particles. Electrorheological fluids (ERFs), where the contact force is due to dielectric contrast, are analogous systems to MRFs in which the particle size effect has been also reported. Shi and Conrad ${ }^{11}$ observed that the yield stress of ERFs increased as particle size was increased in the range $6 \mu \mathrm{m}<d_{p}<100 \mu \mathrm{m}$, where Brownian motion could be fully discarded. Nevertheless, their samples were polydisperse, which obscured the pure effect of particle size. Several works emphasize the role of size polydispersion on the yield stress, where the general finding is that a higher presence of fines causes a notable reduction of the yield stress due to the local weakening of particle chains. ${ }^{14}$ Jun et $a l .{ }^{15}$ observed, however, an enhancement of the yield stress as particle size was increased for ERFs of almost perfectly monodisperse beads in the size range of tens of microns.

While there is a vast number of reports on the yield stress of MRFs and ERFs, studies on the yield stress of MFBs are scarce. Moreover, a standard technique useful to measure the yield stress in MFBs does not exist. To our knowledge yield stress measurements on MFBs have been only reported by Lee. ${ }^{16}$ The technique used by Lee basically consisted of measuring the minimum force needed to pull out a plate from a jammed MFB, ${ }^{16}$ which gave the tangential component of the yield stress. Lee pointed out that a measurable yield stress appeared prominently just at marginal stability and increased monotonically with further increase of the applied field strength. Furthermore, the data suggested that the yield stress depended primarily on the operating conditions, instead of particle size. It must be noted, however, that this conclusion was based on measurements on iron beads of rather large particle size (average particle size between 90 and $275 \mu \mathrm{m}$ ) for which the MFB void fractions were close (between 0.479 and 0.485 ). In the present paper we report on experimental data of the yield stress of MFBs of fine powders (average particle size between 35 and $65 \mu \mathrm{m}$ ) obtained by means of a noninvasive technique. We focus our attention on the dependence of the yield stress on particle size. The results will be rationalized from the effect of particle size on the packing of the MFB, which can be of relevance in the limit of fine particles.

In our experimental setup the powder sample is held in a vertically oriented cylindrical vessel made of polycarbonate (2.54 $\mathrm{cm}$ internal diameter) and rests on a porous plate that acts as gas distributor (5 $\mu \mathrm{m}$ pore size). By means of a series of computer controlled valves and a mass flow controller, a controlled flow of filtered and dried air is pumped through the powder bed while the gas pressure drop $\Delta p$ across it is read from a differential pressure transducer. The height of the bed, which gives an average value of the particle volume fraction $\phi$, is measured by means of an ultrasonic sensor placed on top of the vessel. This device can 


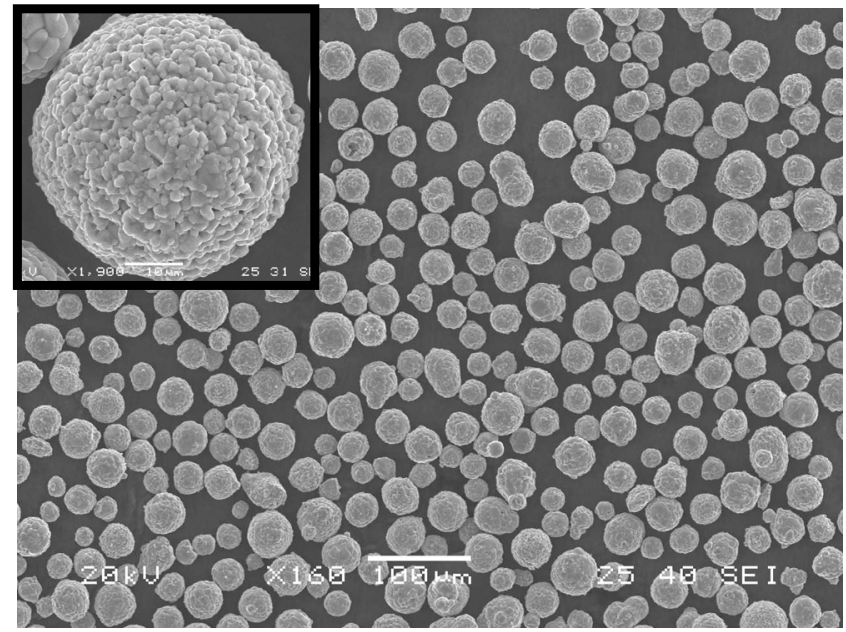

FIG. 2. SEM photograph of $35 \mu \mathrm{m}$ sized magnetite beads used in the experiments.

determine distance, with an accuracy smaller than local fluctuations in bed height, by sending an ultrasonic wave and measuring the time of reflection from the target. Our magnetic powders have been tested as affected by a horizontal uniform magnetic field externally imposed (cross-flow configuration). The strength of the magnetic field is varied by adjusting the electrical current through a pair of square Helmholtz coils $\left(50 \times 50 \mathrm{~cm}^{2}\right)$ with each coil consisting of 500 turns of 2-mm-diam copper wire. The magnetic field strength is measured by a Hirst Magnetics Gaussmeter using an axial probe with an accuracy less than $0.1 \mathrm{mT}$.

The magnetic powders used in the experiments consist of magnetite beads of similar permeability but different particle size $d_{p}(35,50$, and $65 \mu \mathrm{m})$ supplied by Xerox Co. and used as toner carriers in the xerographic process. These beads are formed by sintering of tiny magnetite particles of size of the order of $1 \mu \mathrm{m}$ as can be seen in scanning electron microscopy (SEM) photographs (Fig. 2), which produces a rather monodisperse distribution of spherically shaped beads. We performed a previous work on magnetic characterization of powder samples by means of a superconducting quantum interference device (SQUID) magnetometer (SQUID Quantum Design MPMS XL), where around $1 \mathrm{mg}$ samples were tested. Since particles in the sample were well dispersed the measured susceptibilities can be considered as the material susceptibilities. As expected, initially demagnetized samples behaved linearly in the range of field strengths applied in the MFB experiments described below $(H<5 \mathrm{kA} / \mathrm{m})$ and can be characterized by similar material susceptibilities: $\chi$ $=2.98\left(d_{p}=35 \mu \mathrm{m}\right), \quad \chi=2.78\left(d_{p}=50 \mu \mathrm{m}\right)$, and $\chi=2.60\left(d_{p}\right.$ $=65 \mu \mathrm{m})$.

Figure 3 exemplifies data obtained for the gas pressure drop across the bed $\Delta p$ versus the gas velocity $v_{g}$ for a fixed magnetic field strength and different particle sizes. In the experimental procedure the bed is first initialized by subjecting it to a gas velocity large enough to drive it into a bubbling state, where it looses memory of previous processes. ${ }^{17}$ When the magnetic field is applied, the initial gas velocity must be still sufficiently large to keep the bed in the bubbling regime even in the presence of the magnetic field. Then $v_{g}$ is

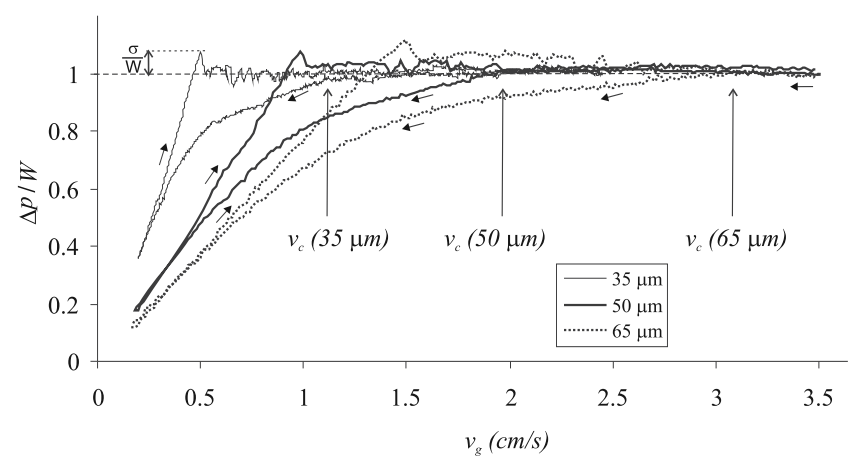

FIG. 3. Gas pressure drop across the powder bed as a function of gas velocity during the fluidization-defluidization cycles in the presence of a magnetic field of strength $H=2.8 \mathrm{kA} / \mathrm{m}$ (made nondimensional with the powder weight per unit area $W$ ). The bed is jammed at $v_{g}=v_{c}$, which is indicated. The pressure overshoot at breaking gives us the tensile yield stress $\sigma$.

slowly decreased while $\Delta p$ is measured. As $v_{g}$ is slowly decreased and the bed is still in the fluidlike bubbling regime, $\Delta p$ balances the powder weight per unit area $W \simeq 510 \mathrm{~Pa}$. The hydrodynamic shear becomes, however, less intense and at a critical gas velocity $v_{c}$ the system is jammed, thus transiting to a solidlike regime because of the prevailing attractive interaction between the particles. Further decrease of $v_{g}$ below $v_{c}$ causes $\Delta p$ to fall below $W$ since part of the weight is sustained by the enduring network of interparticle contacts in the jammed bed.

Gas pressure drop $\Delta p$ measurements allow us to measure the tensile yield stress of the MFB in the solidlike regime when the gas velocity is $v_{g}=v_{0}<v_{c}$. The MFB bed in the solidlike regime is consolidated by a consolidation stress $\sigma_{c}=W-\Delta p_{0}$, where $\Delta p_{0}$ is the gas pressure drop at $v_{0}$ (since we are dealing with shallow beds, wall effects are negligible). If the gas velocity is now increased from $v_{0}$, the solidlike structure remains at first unperturbed and thus $\Delta p$ increases linearly with $v_{g}$ (see Fig. 3), which conforms to Carman's law for the resistance of porous solids to the passage of gas flow. At the so-called point of minimum fluidization velocity, $\Delta p$ balances the powder weight per unit area $W$. At this point a noncohesive bed would loose the solidlike structure, yet $\Delta p$ across the cohesive bed keeps increasing linearly. The gas flow puts the yet solidlike bed under tension, and as the tension builds up there comes a point at which the powder breaks and $\Delta p$ falls down to around $W$ (see Fig. 3). Thus, the pressure overshoot above $W$ gives an estimate of the tensile yield stress $\sigma=\Delta p_{\max }-W$ of the MFB initially consolidated by a consolidation stress $\sigma_{c}$. It is worth noting that this technique enables us to measure the yield stress as affected by the consolidation stress of the powder, which has a great relevance at least in the absence of external magnetic field. ${ }^{18}$ Furthermore, our technique enables us to measure $\sigma$ at states of very low consolidation stress (about $10 \mathrm{~Pa}$ ) near the jamming transition.

Figure 4 shows data on the tensile yield stress $\sigma$ measured in the absence of field and in the presence of applied fields of strengths 1.2 and $3.6 \mathrm{kA} / \mathrm{m}$ as a function of $\sigma_{c}$. The data obtained in the absence of field reproduce the well known result that powder cohesiveness is increased as par- 

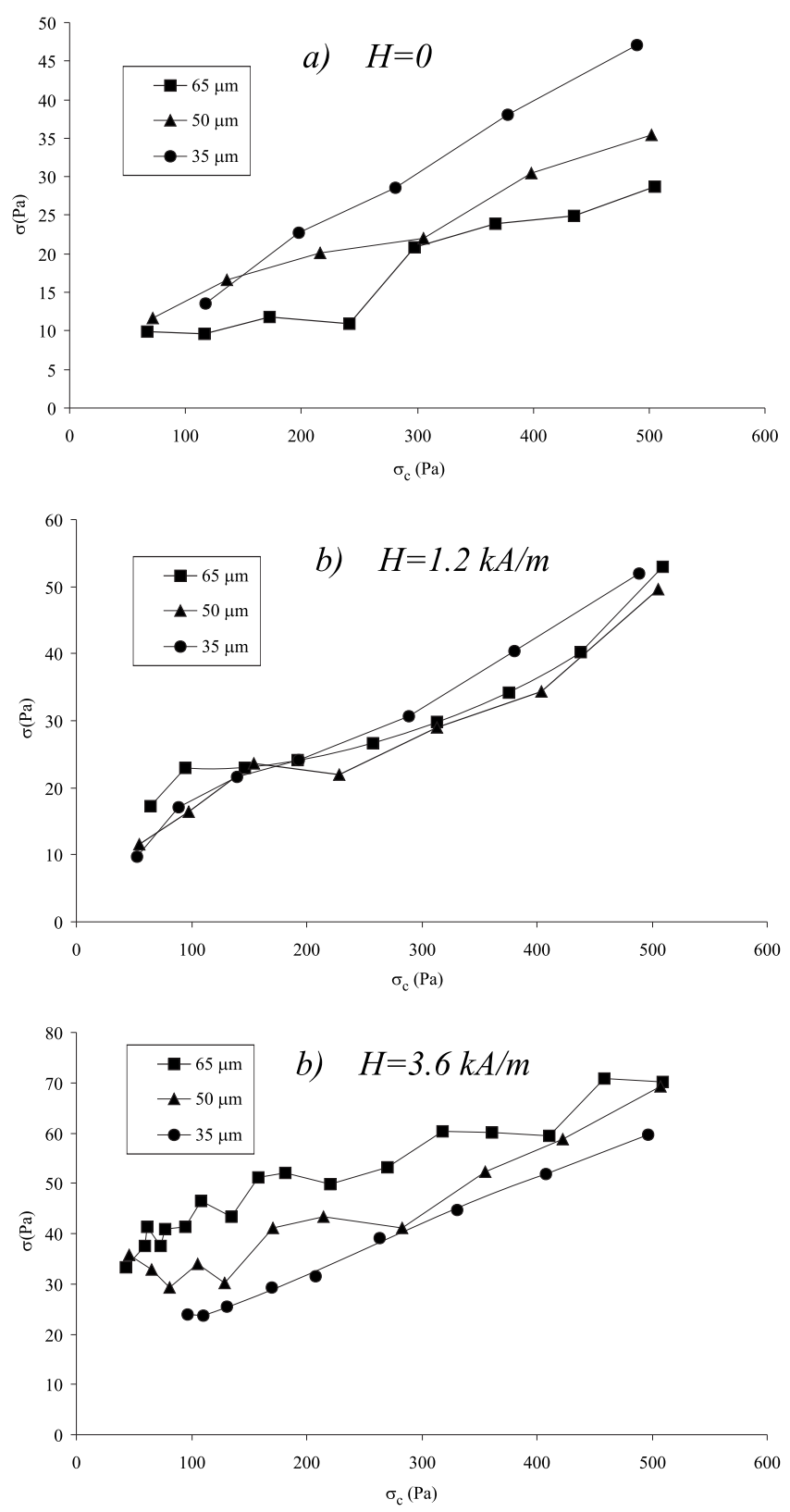

FIG. 4. Measured tensile yield stress as a function of the consolidation stress $\sigma_{c}$ in the (a) absence and presence of applied fields of strength (b) $H=1.2 \mathrm{kA} / \mathrm{m}$ and (c) $H=3.6 \mathrm{kA} / \mathrm{m}$.

ticle size is decreased. Since interparticle attractive force in the absence of field is mainly due to the short-ranged van der Waals force, which is proportional to the typical size of surface asperities, ${ }^{18}$ the tensile yield stress should decrease as bead size is increased. It is also seen that the tensile yield stress increases as $\sigma_{c}$ is increased. This result could be attributable to compaction of the powder but, as it is well documented in the literature of fine cohesive powders, ${ }^{18}$ is primarily due to plastic deformation of the asperities at contact as the consolidation stress is increased. Plastic deformation of interparticle contacts and the consequent enhancement of the interparticle adhesion force are considered in more elaborate theories, such as the Mesarovic and Johnson theory (see Ref. 18 for a detailed review). Note also that $\partial \sigma / \partial \sigma_{c}$ increases as particle size is decreased, which can be explained by the increase of powder compactability as particle size is

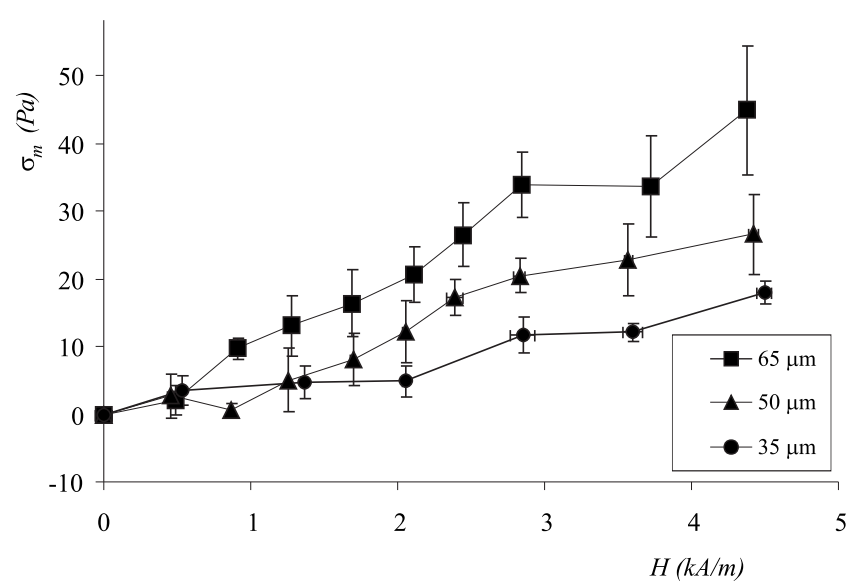

FIG. 5. Magnetic tensile yield stress as a function of the field strength for magnetically stabilized beds of magnetite beads of different size.

decreased. ${ }^{18}$ Let us now analyze the effect of the magnetic field on the yield stress. A remarkable observation from Fig. 4 is that the presence of a field of sufficiently high strength inverts the order of powder cohesiveness, that is the larger the bead size the larger the tensile yield stress of the powder.

Figure 5 shows experimental averaged data of the magnetic stress obtained as $\sigma_{m}=\sigma(H)-\sigma(0)$ as a function of the magnetic field strength $H$. Basically, it is seen that $\sigma_{m}$ increases as particle size is increased as already inferred. Interestingly, the values of the tensile yield stress measured by us are similar to the tangential yield stress $(\tau)$ values measured by Lee ${ }^{16}$ for MFBs operated in the coflow field configuration. For example, Lee reported $10 \mathrm{~Pa}<\tau<30 \mathrm{~Pa}$ for a MFB of iron particles in the size range $74 \mu \mathrm{m}<d_{p}$ $<105 \mu \mathrm{m}, \phi \simeq 0.5$, and $1 \mathrm{kA} / \mathrm{m}<H<4 \mathrm{kA} / \mathrm{m}$, which fits to our data. On the other hand, typical yield stress data reported for MRFs are of the order of $10^{4} \mathrm{~Pa}$ for magnetic fields applied of the order of $100 \mathrm{kA} / \mathrm{m} .{ }^{19-21}$ Even though these data are not directly comparable to our measurements, if we extrapolate our data for similar field strengths by using a quadratic law fit, it can be inferred that the typical yield stress of MFBs would be comparable to the yield stress typically reported for MRFs. Note, however, that the scatter of our measurements does not allow us for a conclusive statement on the scaling of the yield stress with the magnetic field strength.

Let us now look for a possible explanation of the particle size effect observed by us. Figure 6 shows data on the particle volume fraction $\phi$ of the magnetically stabilized bed at jamming $\left(\sigma_{c}=0\right)$ as a function of the magnetic field strength. As can be seen $\phi$ depends on particle size, the smaller the particle size the smaller $\phi$, which can be rationalized on the basis of the increase of the ratio of interparticle magnetic force (scaling proportionally to $d_{p}^{2}$ ) to particle weight (scaling proportionally to $d_{p}^{3}$ ) as particle size is decreased. This is in agreement with the results of Forsyth et al. ${ }^{9}$ on the void fraction of beds of iron beads poured in the presence of a magnetic field. It must be reminded, however, that the magnetic field strength within the bulk of the bed $H_{b}$ will be decreased by demagnetization. Thus, it will be $H_{b}=H-\kappa M$, where $\kappa$ is the demagnetization factor (we will use $\kappa=0.5$, which is the result for an infinite cylinder magnetized trans- 


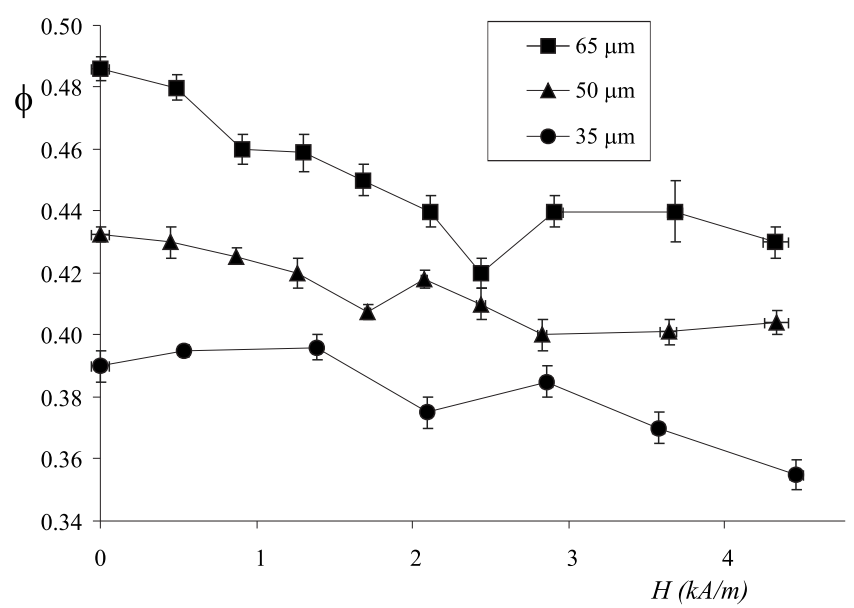

FIG. 6. Particle volume fraction of the magnetofluidized bed at jamming as a function of the strength of the applied magnetic field.

versely to its axis) and $M$ is the bulk magnetization of the bed. In the case of a linearly magnetizable material $H_{b}$ $=H /\left(1+\kappa \chi_{b}\right)$, where $\chi_{b}=M / H_{b}$ is the bulk susceptibility. According to effective medium theories and numerical analysis on random granular materials, ${ }^{22}$ the bulk susceptibility of a bed of magnetically linear spherical particles of susceptibility $\chi$ located in a homogeneous environment of susceptibility $\chi_{0}$ can be calculated from the Bruggeman mixing rule,

$$
(1-\phi) \frac{\chi_{0}-\chi_{b}}{3+\chi_{0}+2 \chi_{b}}+\phi \frac{\chi-\chi_{b}}{3+\chi+2 \chi_{b}}=0 .
$$

In our case $\chi_{0}=0$ and taking into account the variation of $\phi$, it can be estimated that the magnetic field within the bulk of the MFB varies just slightly during our experiments (it is $0.68 \leqq H_{b} / H \leqq 0.72$ in the range from small to large field strengths tested). Moreover it is almost independent of particle size, which rules out its possible role on the particle size effect. On the other hand, the decrease of $\phi$ as particle size is decreased implies that interparticle contacts become on average more vertically oriented, thus the average angle between the contact normal and the horizontal magnetic field $\langle\theta\rangle$ becomes larger as particle size is decreased. As a consequence the interparticle force would be smaller, which would give a smaller yield stress as it is shown by our data. It is remarkable that the yield stress of MFBs can be measured for different orientations of the magnetic field with respect to the pulling force. This can be a useful tool to further explore the effect of physical parameters such as particle size on the magnetic stress. According to our argument, we should observe the opposite size effect in the case that the field is vertically oriented. Experiments on the coflow field configuration are in progress to validate this expectation. Nonetheless, yield stress data on MFBs operated in the coflow field configuration reported by Lee $^{16}$ can be used to check the plausibility of our argument. The results indicated that the yield stress was very sensitive to the variation of $\phi$. In agreement with our reasoning, Lee showed that the yield stresses were increased as $\phi$ was decreased while the rest of parameters were held constant (see Table 4 of Ref. 16). This can be rationalized on the basis that a smaller $\phi$ (higher void fraction) implies on average a smaller angle between the contact normal and the vertical magnetic field, which would result in higher values of the interparticle magnetostatic attraction in the coflow configuration as indicated by Lee's data.

In conclusion, in this work the tensile yield stress of magnetofluidized beds has been measured by means of a noninvasive technique which makes use of the gas flow to put the magnetized bed under tension. Previous fluidization of the sample allows us to obtain an initially reproducible state in which the bed is settled in the presence of the magnetic field and with a controlled consolidation stress. This procedure allows us to discriminate between the magnetic stress and the natural stress arising from van der Waals forces. The natural yield stress (in the absence of an applied magnetic field) is seen to increase as the consolidation stress is increased, which is a well known result reported in the literature of fine cohesive powders. Furthermore, for a given consolidation stress, the yield stress increases as particle size is decreased as expected. This trend is, however, inverted when the magnetic field is applied. As the magnetic field strength is increased the larger particle size sample becomes relatively more cohesive, indicating a significant effect of particle size on the magnetic yield stress. Our work suggests that particle size might have a relevant influence on the yield stress through its effect on the packing arrangement.

This work was supported by Spanish Government Agency Ministerio de Ciencia y Tecnologia (Contract No. FIS2006-03645).

${ }^{1}$ G. Bossis, O. Volkova, S. Lacis, and A. Meunier, Magnetorheology: Fluids, Structures and Rheology, Lectures Notes in Physics Vol. 594, edited by S. Odenbach (Springer-Verlag, Berlin, 2003), pp. 202-230

${ }^{2}$ R. E. Rosensweig, Ferrohydrodynamics (Dover, New York, 1997).

${ }^{3}$ T. B. Jones and B. Saha, J. Appl. Phys. 68, 404 (1990).

${ }^{4}$ Y. Lalatonne, J. Richardi, and M. P. Pileni, Nature Mater. 3, 121 (2004).

${ }^{5}$ D. K. Potter and A. Stephenson, Geophys. Res. Lett. 15, 1097 (1988).

${ }^{6}$ D. J. Klingenberg, Ph.D. thesis, University of Illinois, 1989.

${ }^{7}$ H. Clercx and G. Bossis, Phys. Rev. E 48, 2721 (1993).

${ }^{8}$ S. Lacis, O. Volkova, and G. Bossis, Modelling of Material ProcessingProceedings of the International Colloquium (University of Latvia, Riga, 1999), pp. 222-227.

${ }^{9}$ A. J. Forsyth, S. R. Hutton, C. F. Osborne, and M. J. Rhodes, Phys. Rev. Lett. 87, 244301 (2001).

${ }^{10}$ F. Peters and E. Lemaire, Phys. Rev. E 69, 061302 (2004).

${ }^{11}$ Y. H. Shih and H. Conrad, Int. J. Mod. Phys. B 8, 2835 (1994).

${ }^{12}$ E. Lemaire, A. Meunier, G. Bossis, J. Liu, D. Felt, P. Bashtovoi, and N. Matoussevitch, J. Rheol. 39, 1011 (1995).

${ }^{13}$ B. J. de Gans, N. J. Duin, D. van den Ende, and J. Mellema, J. Chem. Phys. 113, 2032 (2000).

${ }^{14}$ C. W. Wu and H. Conrad, J. Appl. Phys. 83, 3880 (1998).

${ }^{15}$ J.-B. Jun, S.-Y. Uhm, S.-H. Cho, and K.-D. Suh, Langmuir 20, 2429 (2004).

${ }^{16}$ W. K. Lee, AIChE Symp. Ser. 79, 87 (1983).

${ }^{17}$ J. M. Valverde, A. Castellanos, and M. A. S. Quintanilla, Contemp. Phys. 44, 389 (2003).

${ }^{18}$ A. Castellanos, Adv. Phys. 54, 263 (2005).

${ }^{19}$ M. T. Lopez-Lopez, P. Kuzhir, S. Lacis, G. Bossis, F. GonzálezCaballero, and J. D. G. Durán, J. Phys.: Condens. Matter 18, S2803 (2006).

${ }^{20}$ S. Genc and P. P. Phule, Smart Mater. Struct. 11, 140146 (2002).

${ }^{21}$ D. J. Klingenberg, C. K. Olk, M. A. Golden, and J. C. Ulicny, J. Phys.: Conf. Ser. 149, 012063 (2009).

${ }^{22}$ K. Karkkainen, A. Sihvola, and K. Nikoskinen, IEEE Trans. Geosci. Remote Sens. 39, 1013 (2001). 